\title{
Existing PON Infrastructure Supported Hybrid Fiber-Wireless Sensor Networks
}

\author{
Yu, Xianbin; Zhao, Ying; Deng, Lei; Pang, Xiaodan; Tafur Monroy, Idelfonso
}

Published in:

OFC/NFOEC Technical Digest

Publication date:

2012

Document Version

Publisher's PDF, also known as Version of record

Link back to DTU Orbit

Citation $(A P A)$ :

Yu, X., Zhao, Y., Deng, L., Pang, X., \& Tafur Monroy, I. (2012). Existing PON Infrastructure Supported Hybrid Fiber-Wireless Sensor Networks. In OFC/NFOEC Technical Digest Optical Society of America.

\section{General rights}

Copyright and moral rights for the publications made accessible in the public portal are retained by the authors and/or other copyright owners and it is a condition of accessing publications that users recognise and abide by the legal requirements associated with these rights.

- Users may download and print one copy of any publication from the public portal for the purpose of private study or research.

- You may not further distribute the material or use it for any profit-making activity or commercial gain

- You may freely distribute the URL identifying the publication in the public portal

If you believe that this document breaches copyright please contact us providing details, and we will remove access to the work immediately and investigate your claim 


\title{
Existing PON Infrastructure Supported Hybrid Fiber-Wireless Sensor Networks
}

\author{
Xianbin Yu ${ }^{1}$, Ying Zhao ${ }^{1,2}$, Lei Deng ${ }^{1,3}$, Xiaodan Pang ${ }^{1}$, Idelfonso Tafur Monroy ${ }^{1}$ \\ ${ }^{1}$ DTU Fotonik, Technical University of Denmark, DK-2800, Kgs. Lyngby, Denmark. E-mail: xiyu@fotonik.dtu.dk \\ ${ }^{2}$ Department of Electronic Engineering, Tsinghua University, 100084 Beijing, China. \\ ${ }^{3}$ School of Optoelectronics Science \& Engineering, HuaZhong University of Science and Technology, Wuhan, China.
}

\begin{abstract}
We propose a hybrid fiber wireless sensor network based on the existing PON infrastructure. The feasibility of remote sensing and PON convergence is experimentally proven by transmitting direct-sequence spread-spectrum wireless sensing and 2.5Gbps GPON signals.

OCIS codes: (120.0280) Remote sensing and sensors; (060.5625) Radio frequency photonics, (060.4254)

Networks, combinatorial network design
\end{abstract}

\section{Introduction}

The advancements in wireless communications have enabled the rapid development of wireless sensor networks (WSN) [1], which have a very wide range of applications e.g. in monitoring temperature, strain, pressure etc. However, due to the low wireless emission power and low cost requirementswhich are important features of sensor networks- the sensor manager who collects and decodes the sensing information in a traditional WSN, has to be located closely to the region of interest. The development of a remote sensing network which can monitor remote or inaccessible regions, or in seriously life threatening environments like chemical radioactivity, disaster observation, E-health monitoring [2, 3], is highly desirable. Facing this challenge, the integration of optical-wireless communication networks provides a promising solution [4], since optical transmission has the advantages of large capacity to support huge number of sensor nodes if needed, low attenuation, extended reach and quality of service (QoS) support [5].

We propose an architecture as depicted in Fig. 1 for a hybrid fiber wireless sensor network based on the existing gigabit passive optical networks (GPON) infrastructure. In this scenario, each head sensor node is responsible for collecting sensing information from all sensor nodes within its cluster. A single principal head sensor node performs multi-functionally as an interface to the optical fiber based PON, to convert all the received sensing signals into the optical domain, including the forwarded from the other head sensor nodes. After the optical transmission over the PON, the sensor task manager remotely processes sensing information and thereby observes the sensing region.

To the best of our knowledge, the research on hybrid fiber-wireless sensor networks in the physical layer is still at an early stage with so far limited published work on implementation requirements and more importantly integration into PON architectures. There are reports either on architecture design/simulation in the network layer [6], experimental demo for indoor positioning by employing an infrared lightwave [7], or high speed on-off keying communications for inside aircraft monitoring [8]. The potential of integration of WSN with the existing fiber based PON, in terms of modulation format, radio frequency (RF) efficiency, co-propagation crosstalk, power consumption and cost issue in the physical layer, remains significantly unexplored.

In this paper, we propose a hybrid fiber wireless sensor network by exploiting the current GPON infrastructure and introdcuing a wireless sensor network overlay, to realize remote sensing. A physical link experiment based on code division multiple access (CDMA) technique is investigated to support efficient RF reuse and high sensor node density. Moreover, the performance of both GPON and sensing signals are analyzed and the convergence feasibility of WSN and GPON is also discussed.

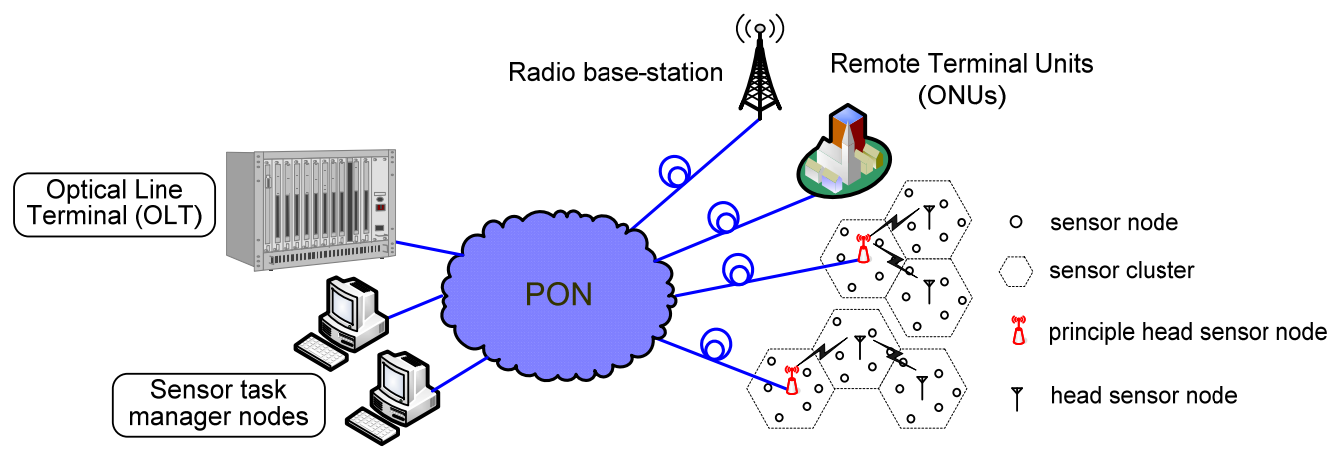

Fig. 1. A hybrid fiber-wireless sensor network based on the existing converged wired/wireless PON. 

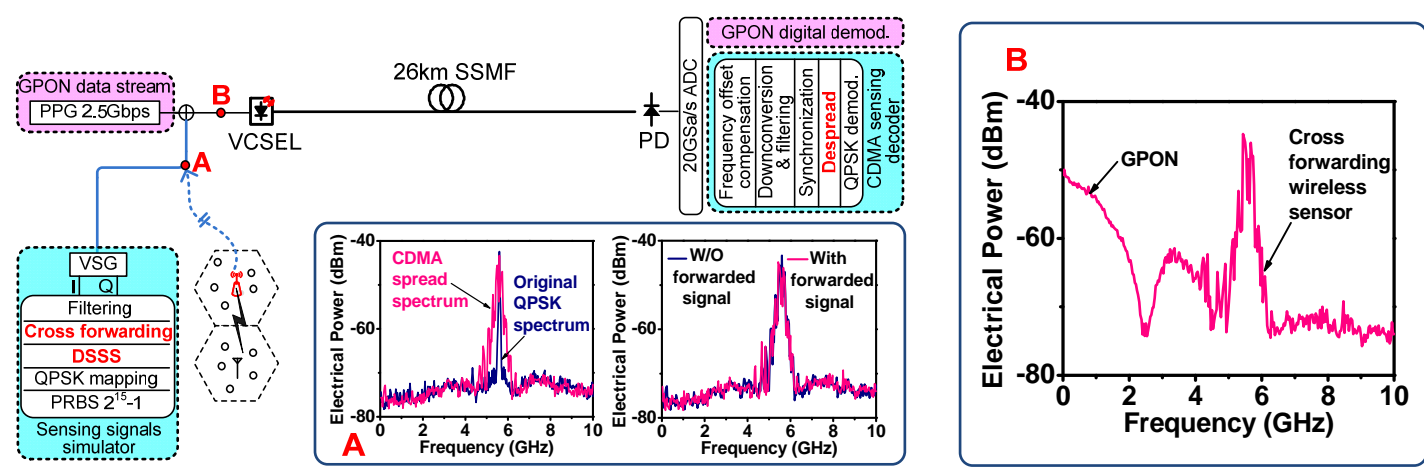

Fig. 2. (a) Experimental setup for convergence fiber transmission of GPON and sensing signals. (A) Spectra of CDMA and original QPSK signals (left) and spectra at the principle head sensor node with/without forwarded signal (right). (B) Spectra of converged 2.5Gbps GPON and 2-cluster sensing signals. PPG: pulse pattern generator. VCSEL: vertical-cavity surfaceemitting laser. SSMF: standard single mode fiber. VSG: vector signal generator. PD: photodiode.

\section{Experimental setup and sensing simulator}

Fig. 2(a) shows the proof-of-concept experimental setup for hybrid fiber wireless sensor networks. A single channel fiber distribution system for GPON and sensing signals is built for the demonstrate purpose. In the transmitter, a vector signal generator (VSG) is used to emulate the wireless sensing signal received by the principal head sensor node, which interfaces to an optical fiber link. The principal head sensor node collects the sensing signal within the cluster, and as well as forward the sensing signal from other head nodes. The RF carrier for sensing is set at $5.6 \mathrm{GHz}$, since the radio location band of $5.47-5.85 \mathrm{GHz}$ has been widely suggested for industrial, scientific and medical (ISM) applications. The VSG is I/Q modulated by a baseband waveform which is digitally generated from a 1.25GSa/s arbitrary waveform generator (AWG).

To effectively reuse RF frequency resource and support high node-density in one sensor cluster, code division multiple access (CDMA) technique is adopted in our system. The baseband signal is generated under a direct-sequence spread-spectrum (DSSS) modem architecture by using an offline digital signal processing (DSP) sensing signal simulator. In the digital domain, a pseudo random binary sequence (PRBS) with a length of $2^{15}-1$ is generated and mapped to form a 78.125Baud/s quadrature phase shift keying (QPSK) symbol sequence. The 8-bit orthogonal Walsh code to perform node identification is multiplied by each symbol of QPSK sequence. Therefore, the DSSS chip sequence with a chip rate of $78.125 \times 8=625 \mathrm{chip} / \mathrm{s}$ is obtained in parallel for 8 nodes in one cluster. After spectrum spreading, the cooperation between head nodes is digitally emulated in the delay and overlap module, taking into account the wireless routing loss of 2.2dB. The output is then filtered and upconverted to $5.6 \mathrm{GHz}$. The spectra of original QPSK and DSSS sequences are measured at the point A and shown in Fig.2 (A). The right inset figure in Fig.2 (A) displays the sensing signal spectra measured at the point A for two cases: one cluster with only principle head sensor node and 2-cluster with co-existence of one principle and one forwarded head sensor nodes.

In addition, 2.5Gbps GPON data stream is generated from a pulse pattern generator (PPG) for an end-to-end GPON link. A converged GPON/sensing signal, as shown in Fig. 2 (B) measured at the point $\mathrm{B}$, is then modulated onto an optical carrier at $1540.68 \mathrm{~nm}$ emitted from a commercially available directly modulated vertical-cavity surface-emitting laser (VCSEL). After a 26km fiber transmission and direct detection, the converged signal is digitalized by a 20GSa/s analog-to-digital converter (ADC) and separately decoded by two digital signal processing (DSP) receivers in the digital domain. For the wireless sensor channel, chip sequence dispreading is performed in the CDMA sensing decoder to identify different nodes, after frequency offset compensation and down conversion. The bit error rate (BER) is subsequently evaluated by direct error counting of $3 \times 10^{4}$ bits.

\section{Experimental results and discussions}

In our experiment, we measure the BER performances for both GPON data and sensing signal. Fig. 3(a) shows the BER as a function of the received optical power before the photodiode (PD) for 2.5Gbps GPON data, with and without the co-propagation of wireless sensing signal. It can be seen that in both back to back (B2B) and $26 \mathrm{~km}$ SSMF transmission cases, the power penalty induced by emerging the wireless sensor signals into the GPON systems is negligible, which proves the feasibility of convergence of the existing optical GPON with wireless sensor networks. On the other hand, due to 
the undesired chirp of the VCSEL, the $26 \mathrm{~km}$ fiber dispersion introduces $~ 2 \mathrm{~dB}$ power penalty for 2.5Gbps GPON data.

Fig. 3(b) presents the BER performances of the sensing signals, as well as with and without copropagation of GPON signals. In this case, only one sensor cluster is supported by the principal head node without any other forwarded sensing signals. Similarly, we can see that the quality of the wireless sensing signals has negligible degradation caused by the 2.5Gbps GPON data both in B2B and after 26 $\mathrm{km}$ SMF transmission. In the mean time, a penalty of $\sim 2.5 \mathrm{~dB}$ jointly introduced by the fiber dispersion and chirp is observed for the wireless sensing signals. Therefore, from the wireless networks side, the possibility of the remote fiber wireless sensing realization based on a current 2.5Gbps GPON infrastructure is confirmed.

The BER performance of the forwarded sensing signal in the 2-cluster scenario is shown in Fig. 3(c), with the co-propagation of $2.5 \mathrm{Gbps}$ GPON data. Very similarly, the $26 \mathrm{~km}$ fiber transmission also causes a $2.5 \mathrm{~dB}$ penalty to the hybrid multi-cluster system. Furthermore, by comparing Fig.3(b) and (c), it can be seen that the BER performances of two head nodes in two clusters are almost the same for both B2B and $26 \mathrm{~km}$ fiber transmission. This result implies that the wireless relay in wireless sensor networks introduces no extra penalty between the principal sensor cluster and the remote sensor cluster. Therefore, multi-cluster wireless sensing is also firmly supported in this hybrid fiber wireless sensor networks, while maintaining the same transmission performances for all of them.

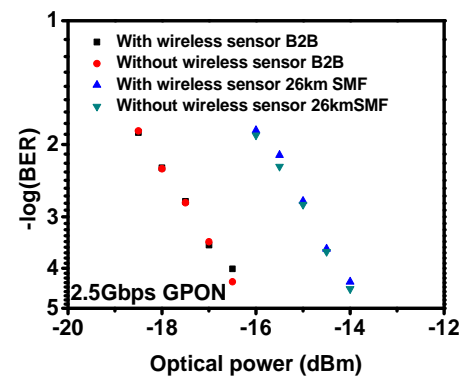

(a)

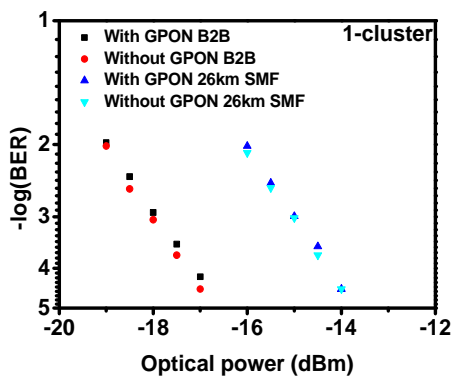

(b)

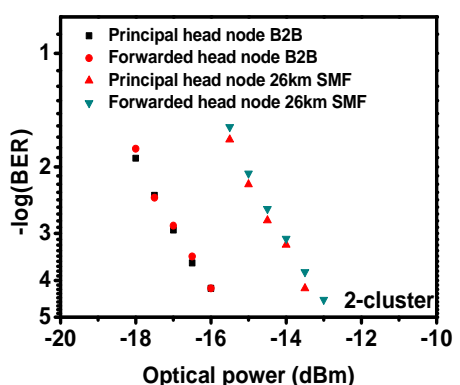

(c)

Fig. 3. Measured BER performances of (a) 2.5Gbps GPON signal, (b) one cluster with only principle head sensor node, and (c) 2-cluster with co-existence of one principle and one forwarded head sensor nodes.

\section{Conclusions}

We propose a hybrid fiber wireless sensor network supported by the existing optical fiber PON infrastructure. We demonstrate converged fiber transmission of multi-cluster sensing and 2.5Gbps GPON signals. In the experiment, direct-sequence spread spectrum based CDMA technique is employed to support efficient RF reuse and high sensor node density. The experimental results verify the feasibility to integrate wireless sensor networks into the existing GPON with negligible performance degradation. Our results shows the potential re-use of the PON infrastructure for hybrid fiber wireless sensor networks to realize a large number of remote sensing applications in inaccessible regions, life hazardous environment or disaster areas.

\section{References}

[1] I. F. Akyildiz, W. Su, Y. Sankarasubramaniam, and E. Cayirci, “A survey on sensor networks,” IEEE Communications Magazine, vol. 40, no. 8, pp.102-114, August 2002.

[2] V. A. Kottapalli, A. S. Kiremidjian, J. P. Lynch, E. Carryer, T. W. Kenny, K. H. Law, and Y. Lei, "Two-tiered wireless sensor network architecture for structural health monitoring," SPIE 10th Annual International Symposium on Smart Structures and Materials, March 2003.

[3] W.-Y. Chung, G. Walia, "Query driven architecture in wireless sensor network for ubiquitous health monitoring," Sensor Letters, vol. 9, no. 1, Feb. 2011, pp. 384-392.

[4] C. Qiao, J. Wang, T. Wang, "Integrated optical wireless access/metro networks”, Invited paper, OFC/NFOEC, March 2125, 2010, San Diego, USA.

[5] X. Yu, J. B. Jensen, D. Zibar, C. Peucheret, I. T. Monroy, "Converged wireless and wireline access system based on optical phase modulation for both radio-over-fiber and baseband signals,” IEEE Photon. Technol. Lett., vol. 20, no. 21, pp. 18141816, Nov. 2008.

[6] J. Tang; X. Jin; Y. Zhang; X. Zhang; W. Cai; “A hybrid radio over fiber wireless sensor network architecture,” 2007 International Conference on Wireless Communications, Networking and Mobile Computing, WiCom 2007. pp. 2675-2678.

[7] J. Fadlullah andM. Kavehrad, "Indoor high-bandwidth optical wireless links for sensor networks," J. Lightw. Technol., vol. 28, no. 21, pp. 3086-3094, Nov. 1, 2010.

[8] W. Jian, A. Chowdhury, Z. Jia, C. Estevez, and G.-K. Chang, "Energy-efficient multi-access technologies for very high throughput avionic millimetre-wave, wireless sensor communication networks source,” J. Lightw. Technol., vol. 28, no. 16, pp. 2398-2405, 2010. 